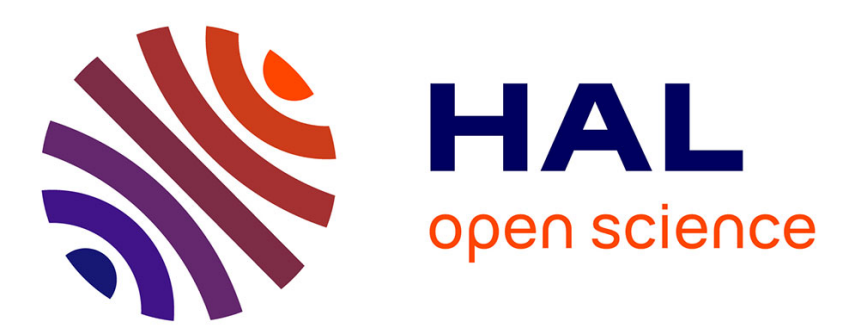

\title{
Band structure parameters of metallic diamond from angle-resolved photoemission spectroscopy
}

H. Guyot, P. Achatz, A Nicolaou, P Le Fèvre, F Bertran, A Taleb-Ibrahimi, E. Bustarret

\section{- To cite this version:}

H. Guyot, P. Achatz, A Nicolaou, P Le Fèvre, F Bertran, et al.. Band structure parameters of metallic diamond from angle-resolved photoemission spectroscopy. Physical Review B: Condensed Matter and Materials Physics (1998-2015), 2015, 92 (4), pp.045135. 10.1103/PhysRevB.92.045135 . hal-01218889

\section{HAL Id: hal-01218889 \\ https://hal.science/hal-01218889}

Submitted on 21 Oct 2015

HAL is a multi-disciplinary open access archive for the deposit and dissemination of scientific research documents, whether they are published or not. The documents may come from teaching and research institutions in France or abroad, or from public or private research centers.
L'archive ouverte pluridisciplinaire HAL, est destinée au dépôt et à la diffusion de documents scientifiques de niveau recherche, publiés ou non, émanant des établissements d'enseignement et de recherche français ou étrangers, des laboratoires publics ou privés. 


\title{
Band structure parameters of metallic diamond from angle-resolved photoemission spectroscopy
}

\author{
H. Guyot, ${ }^{1,2,{ }^{*}}$ P. Achatz, ${ }^{1,2}$ A. Nicolaou, ${ }^{3}$ P. Le Fèvre, ${ }^{3}$ F. Bertran, ${ }^{3}$ A. Taleb-Ibrahimi, ${ }^{3}$ and E. Bustarret ${ }^{1,2}$ \\ ${ }^{1}$ Université Grenoble Alpes, F-38000 Grenoble, France \\ ${ }^{2}$ CNRS, Institut NEEL, F-38042 Grenoble, France \\ ${ }^{3}$ Synchrotron SOLEIL, BP 48, F-91192 Gif-sur-Yvette Cedex, France \\ (Received 14 April 2015; revised manuscript received 2 July 2015; published 31 July 2015)
}

\begin{abstract}
The electronic band structure of heavily boron doped diamond was investigated by angle-resolved photoemission spectroscopy on (100)-oriented epilayers. A unique set of Luttinger parameters was deduced from a comparison of the experimental band structure of metallic diamond along the $\Delta(\Gamma X)$ and $\Sigma(\Gamma K)$ high-symmetry directions of the reciprocal space, with theoretical band structure calculations performed both within the local density approximation and by an analytical k·p approach. In this way, we were able to describe the experimental band structure over a large three-dimensional region of the reciprocal space and to estimate hole effective masses in agreement with previous theoretical and experimental papers.
\end{abstract}

DOI: 10.1103/PhysRevB.92.045135

PACS number(s): 79.60.Dp, 71.20.-b, 71.15.Dx

\section{INTRODUCTION}

Diamond was recognized long ago [1] as an extreme case of an elemental cubic semiconductor, well suited for testing both the accuracy of electronic band structure calculations, at that time through a Hartree-Fock approach [2,3], and the potential of photoemission experiments to access not only the surface but also the bulk electronic structure. Although this crystal has been studied for more than 30 years, the number of experimental papers on this archetypical covalent material using state-of-the-art, high-resolution, angle-resolved photoemission spectroscopy (ARPES) remains rather poor. After the pioneering experiments of Himpsel et al. [4], who performed energy-dependent photoemission spectroscopy to determine its three-dimensional (3D) electronic structure [5], and the theoretical investigation of Chelikowsky and Louie by first principles methods [6], the following papers dealt mostly with surface properties [7-9] for the main surface orientations and reconstructions, except for one paper on a (111)-oriented diamond at fixed initial state or fixed final state energies [10]. More recently, the interest of the scientific community focused on heavily boron doped epilayers in connection to their metallic and superconductive properties [11,12]. Consequently, high-resolution ARPES experiments have been performed on (111)-oriented heavily boron doped epilayers in order to determine the bulk valence electronic structure [13-15]. Except for a small rigid energy shift of the electronic bands, the first of these papers revealed that diamond crystals, doped slightly below the critical boron concentration for metallicity, conserve the same electronic structure as the metallic ones and that the only effect of the boron doping is a simple shift of the electronic bands [13]. To our knowledge, ARPES studies of (100)-oriented single crystals or epilayers displaying these properties have not been reported yet and the experiments presented below aim at filling this gap. Moreover, although it is well established that spin-orbit coupling is weak in diamond [16-18], its consequences on the electronic structure near the Fermi level $\left(E_{F}\right)$ are still debated. In particular, the value of the splitting at the top of the valence band has been the

*Corresponding author: herve.guyot@neel.cnrs.fr subject of a controversy [19-22]. More generally, the values of the Luttinger parameters for the warped electronic structure around the $\Gamma$ point are still the object of debate. Similar to the case of spin-orbit splitting [22], the various experimental probes (mostly optical spectroscopy or temperature-dependent magnetotransport) do not measure these parameters directly but rather measure an ensemble of many different low-energy excitations, which might not be determined by the sole band structure. For example, in $p$-type diamond, the optically detected energies of the impurity-related excited states may not be even roughly representative of the host crystal band structure [23]. Furthermore, similar experimental probes do not always yield similar results [24]. Recently, pristine diamond of improved quality, where interesting magnetotransport properties may be observed, has been the object of theoretical [25] and experimental [26] papers. It is in this context that high-resolution ARPES measurements are necessary for a complete understanding of the electronic structure of this system and to connect it to low-energy electronic excitations.

Warped band structures have long been described by the popular k.p model proposed early on for many semiconductors $[27,28]$ and by its simplified expression in which spin-orbit interactions may be neglected [29]. The $\mathbf{k} \cdot \mathbf{p}$ model describes the nonisotropic electronic bands as quartic and parabolic along the principal axes and it is therefore generally not applicable throughout the whole Brillouin zone (BZ). However, close to a band extremum, here the valence band maximum at $\Gamma$, it yields the band structure in any $\mathbf{k}$ point of the reciprocal space. While the valence band top features at $\Gamma$ were generally investigated in semiconducting $p$-type diamond by the optical and magnetotransport measurement mentioned above, the limits of the k.p description farther from the zone center have not been confronted in experiments. We perform such a comparison in the present paper. We also tackle this question by adjusting the parameters of the $\mathbf{k} \cdot \mathbf{p}$ analytical description in order to fit rescaled results of first principles or $a b$ initio numerical calculations, at least along the principal directions of the BZs $\Delta$ and $\Sigma$, in the spirit of a previous paper [30] using the linear muffin tin orbital (LMTO) approximation.

Since the discovery of superconductivity in diamond $[11,12]$, numerous $a b$ initio calculations of the valence band structure of boron doped and undoped diamond have 
been published. Although it soon became clear that both optical phonon modes and resonant substitutional boron-related modes contribute to the electron-phonon coupling, which is responsible for hole pairing at low temperatures [31-33], it was generally concluded that when the periodic doping artifacts were removed, for example, using the coherent potential approximation (CPA) [34,35], the valence band parameters remained essentially the same upon boron doping. This led us to compare our photoemission data with $a b$ initio calculations for an undoped diamond, while imposing a rigid shift of the calculated bands in order to account for the different doping, similarly to previous papers $[13,14]$. In the context of the discussion of experimental photoemission results, however, the results of the CPA papers mentioned earlier [34,35], which included chemical doping and disorder, should also be kept in mind. Finally, none of these theoretical approaches have considered the in-plane biaxial strain effects [36] known to be present in most heavily doped diamond epilayers [37,38].

This paper is organized as follows: Sec. II describes experimental and computational methods that were used to obtain the results presented and discussed in Sec. III. The comparison of the experimental dispersion curves with theoretical calculations is also made in Sec. III. The comparison of the resulting values for the band parameters with previous papers is presented and discussed in Sec. IV. Summarizing remarks and conclusions are presented in Sec. V.

\section{EXPERIMENTAL AND COMPUTATIONAL DETAILS}

Various heavily boron doped diamond samples $\left(3 \times 3 \mathrm{~mm}^{2}\right)$ were prepared in a vertical silica tube reactor by microwave plasma-enhanced chemical vapor deposition (MPCVD) of methane and diborane diluted in ultrapure hydrogen [39]. The total pressure and flow rate were 30 torr and $100 \mathrm{sccm}$, respectively, and the substrate temperature was estimated at $830^{\circ} \mathrm{C}$ with a monochromatic pyrometer. The surface of the type Ib substrates was oriented along (001) with a miscut angle below $1^{\circ}$, and the methane-to-hydrogen molar ratio was $4 \%$. The experimental results detailed below were obtained on a $4 \mu \mathrm{m}$ thick epilayer (\#PA15) grown at a boron-to-carbon molar ratio in the gas phase of $2000 \mathrm{ppm}$, resulting in a boron atomic density close to the surface equal to $7 \times 10^{20} \mathrm{~cm}^{-3}$, which is twice lower than that of the bulk according to calibrated secondary ion mass spectrometry (SIMS) measurements. This sample has been shown to become superconducting under a critical temperature of $1.7 \mathrm{~K}$ [39]. These results were found to be typical of those obtained on other epilayers grown under similar conditions along the same crystallographic orientation.

Before their introduction into the ultrahigh vacuum (UHV) preparation chamber of the photoemission setup, where they were UHV annealed, the samples were chemically cleaned in a liquid mixture of three concentrated acids $\left(\mathrm{HNO}_{3}+\mathrm{H}_{2} \mathrm{SO}_{4}+\right.$ $\mathrm{HClO}_{4}$ ) maintained at $80^{\circ} \mathrm{C}$ for $2 \mathrm{~h}$. The annealing under UHV was undertaken around $420^{\circ} \mathrm{C}$, high enough for removing adsorbed contaminants from the diamond surface and low enough to prevent the surface reconstruction observed at higher temperatures on pure diamond crystal surfaces [5]. We did not observe any sign of electronic surface states. In order to perform ARPES measurements, the sample was mounted on a holder that could only be rotated around a vertical axis, which was perpendicular to the plane of incidence containing the photon beam and the normal to the sample surface. Additional $45^{\circ}$ rotations of the sample around an axis perpendicular to its surface were performed ex situ. ARPES measurements were performed at $10 \mathrm{~K}$ in order to reduce the thermal broadening of the quasiparticles and to better resolve the different electronic bands. The overall energy resolution, estimated by the fit of the Fermi level with a Fermi function, was set to 220 and 290 meV using excitation energies of 170 and $402 \mathrm{eV}$, respectively.

The ARPES measurements were carried out on the CASSIOPEE beamline of the synchrotron SOLEIL using an R4000 hemispherical analyzer from Scienta. The entrance slit was orthogonal to the plane of incidence.

For ARPES one shines a monochromatic beam on a solid and measures the kinetic energies $E_{\text {kin }}$ and the takeoff angle $\theta$ with respect to the sample surface of the electrons that are emitted by photoelectric effect. Applying the energy and momentum conservation laws allows us to retrieve the binding energy and the wave vector $\mathbf{k}$ of the electrons in the solid [40], as presented in Eq. (1), where $\hbar$ is the Plank constant, $m$ is the electron mass, $V_{0}$ is the inner potential, and $\mathbf{k}_{\perp}$ and $\mathbf{k}_{\|}$are the out-of-plane and in-plane wave vectors, respectively:

$$
\begin{aligned}
& \mathbf{k}_{\|}=\frac{\sqrt{2 m}}{\hbar} \sqrt{E_{\mathrm{kin}}} \sin \theta \\
& \mathbf{k}_{\perp}=\frac{\sqrt{2 m}}{\hbar} \sqrt{E_{\mathrm{kin}}+V_{0}-\frac{\hbar^{2}}{2 m}} k_{\|}^{2}
\end{aligned}
$$

The component of $\mathbf{k}$ that is parallel to the sample's surface, $\mathbf{k}_{\|}$, is conserved in the photoemission process, and it is only a function of the kinetic energy and the emission angle. As for the component of $\mathbf{k}$ perpendicular to the sample surface, $\mathbf{k}_{\perp}$, it is not conserved due to the broken periodicity at the surfacevacuum interface. In this case, $\mathbf{k}_{\perp}$ varies monotonically with energy, but it is also function of the inner potential $V_{0}$ that is not known a priori. In order to find the inner potential $V_{0}$, which is necessary to relate the excitation energy to the value of $\mathbf{k}_{\perp}$, we performed extended energy dependencies over the second and third BZs with an energy step of $5 \mathrm{eV}$. The centers of these BZs are reached for the energies 172 and $402 \mathrm{eV}$, respectively. Using an inner potential $V_{0}=17.7 \mathrm{eV}$, the centers are located at $\Gamma_{002}=(0,0,7.10) \AA^{-1}$ and $\Gamma_{003}=$ $(0,0,10.48) \AA^{-1}$, respectively.

The polarization of the photons was alternatively selected linear horizontal (HP) or linear vertical (VP) in order to detect orbitals with different symmetry. Since the band structure mappings appear more contrasted in the HP configuration, the ARPES results presented in this paper are limited to this configuration.

In addition to the measurement of the experimental band structure, a theoretical approach has been developed. Beside theoretical bands calculated using an analytical k·p model along several directions of the reciprocal space, a selfconsistent $a b$ initio calculation based on the density functional theory (DFT) within the local density approximation (LDA) was also run to provide more realistic valence band structures [41] along two high-symmetry directions, the $\Gamma X$ and $\Gamma K$ axes, respectively labeled $\Delta$ and $\Sigma$. Such band structures have long been described by the popular k·p model, as proposed early on for semiconductors [27]. This analytical 
model is based on a set of parameters directly linked to the Luttinger parameters [42] $\gamma_{1}, \gamma_{2}$, and $\gamma_{3}$, the warping being directly proportional to the difference between $\gamma_{2}$ and $\gamma_{3}$. The fit of the ARPES bands by this model over a relatively wide energy range (here $4 \mathrm{eV}$ ) may provide an experimental determination of these parameters. Closer to their maximum, the bands may also be considered as parabolic, and effective masses may be defined along the principal axes. However, because of the nonparabolicity, the values deduced from experimental dispersion curves depend strongly on the chosen energy range. This dependence may be removed by adding an additional term $\alpha$ to the dispersion relationship, which becomes:

$$
\mathrm{E}(\mathbf{k})=\mathrm{E}\left(\Gamma_{25}^{\mathrm{v}}\right)-\left(\hbar / 2 m_{0} m^{*}\right) \mathbf{k}^{2}\left(1-\alpha \mathbf{k}^{2}\right),
$$

where $\Gamma_{25}^{\mathrm{v}}$ refers to the top of the band according to the notation of Dresselhaus et al. [27].

Effective masses along the principal axes may also be expressed as a function of $\gamma_{1}, \gamma_{2}$, and $\gamma_{3}$ determined over the same energy range [29,30]. In this paper, effective masses deduced from both $\mathbf{k} \cdot \mathbf{p}$ and DFT calculations will be compared to the values available in the literature.

\section{EXPERIMENTAL AND COMPUTATIONAL RESULTS}

\section{A. High-symmetry directions}

The doped diamond epilayer was first studied using HP photons at $402 \mathrm{eV}$ [Fig. 1(a)] and $170 \mathrm{eV}$ [Fig. 1(b)] in order to probe the $3 \mathrm{D}$ band structure along the $\Delta$ high-symmetry direction in the third and second BZs, respectively. The valence band dispersion was measured down to $8 \mathrm{eV}$ below the Fermi level. Two holelike electronic bands centered at $\Gamma$ and merging at the zone center are clearly seen. In the case of the third BZ [Fig. 1(a)], the measurement extended from the zone center $\Gamma$ to the zone boundary $X$, whereas the momentum range was slightly narrower in the second BZ.

The ab initio DFT calculations for pure diamond along this same direction led to one twofold degenerate band $\left(\Gamma_{25}^{\mathrm{v}} X_{4}\right)$ and another more dispersive $\left(\Gamma_{25}^{\mathrm{v}} X_{1}\right)$ branch. In order to match the experimental data, the calculated bands had to be offset by $2.75 \mathrm{eV}$ and then stretched in energy by a coefficient of 1.23. Such a renormalization of the bandwidth was also used previously when comparing linear combination of atomic orbitals (LCAO) calculations to photoemission spectra of undoped diamond surfaces [5] and is usually attributed to the insufficient description of electron-electron interactions by LDA-DFT simulations [43]. As expected, this scaling coefficient is larger for the low $\mathrm{Z}$ number carbon diamond lattice than for its $\mathrm{Si}, \mathrm{Ge}$, and Sn crystalline counterparts $[43,44]$. These results confirm that the band structure measured by ARPES on heavily boron doped diamond epilayers grown along (100) closely resembles that expected for undoped bulk diamond, similar to what has been observed in the fourth BZ at 825 [13] and $865 \mathrm{eV}$ [14] on (111)-oriented free standing diamond epilayers. The bulksensitive band structures of diamond are therefore detected over a large range of photon energies starting from mid x rays, as published recently, down to ultraviolet photons used in the 1980s for studying hydrogenated surface reconstructions.

To access the $\Sigma$ direction, as presented in Fig. 2(a), the sample was rotated by $45^{\circ}$ around its normal to the surface.
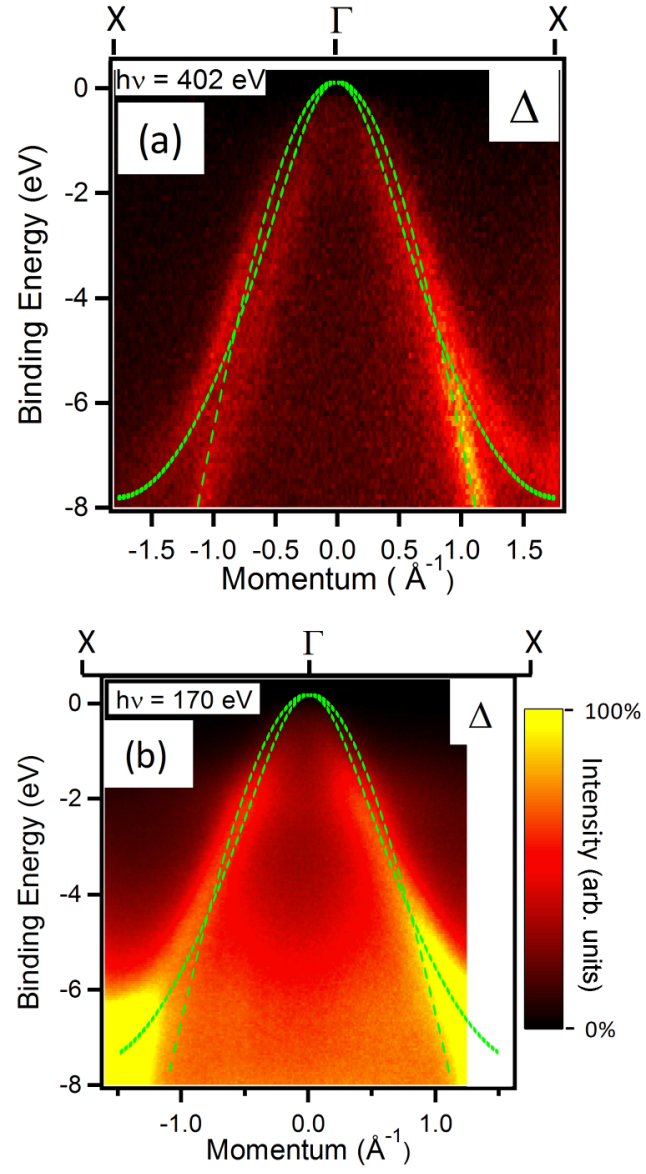

FIG. 1. (Color online) Mapping of the $\Gamma X$ band structure of metallic diamond, measured (a) in the third or (b) in the second BZ. Corrected DFT bands (green dashed lines) are superimposed.

The three bands degenerate at $\Gamma$ expected from the DFT calculations were clearly observed, and from the comparison with band structure calculations, we have estimated the position of the band top to be $150 \pm 50 \mathrm{meV}$ above $E_{F}$, consistent with the metallic character of this sample.

This is illustrated by Fig. 2(b), where momentum distribution curves (MDCs) corresponding to intensity profiles at a constant binding energy (varying by steps of $0.2 \mathrm{eV}$ from 0 to $-4 \mathrm{eV}$ ) were stacked in such a way that the three components and their dispersion become quite apparent. A numerical decomposition of these profiles with three Gaussian components yielded the experimental points that have been superimposed to the intensity mapping in Figs. 2(a) and 2(b). Similar to the $\Delta$ direction, the DFT-LDA calculations results, offset and stretched by the quantities defined above, are shown in Fig. 2(a) to be in good agreement with the experimental band dispersion curves along the $\Sigma$ axis.

It would be natural to assign the less dispersive band to "heavy holes" (HHs) and the two other components to "light holes" (LHs). However, the energy dispersion will not be the same depending on the momentum direction, so the same band will consist of LHs along one direction (e.g., parallel to $\Delta$ ) and HHs for another (e.g., parallel to $\Sigma$ ). The k·p model readily reproduces this "warped" structure of the valence band of diamond, as illustrated in Fig. 3, where a unique 

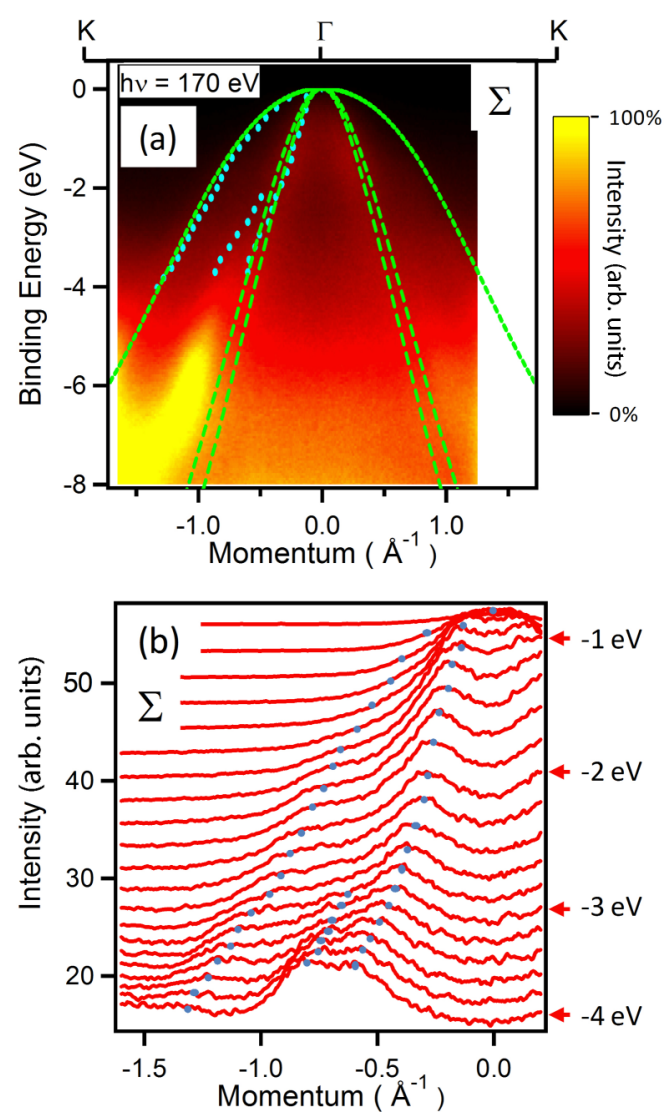

FIG. 2. (Color online) (a) Mapping of the $\Gamma K$ band structure of metallic diamond measured in the second BZ. The blue cyan dots represent the dispersing structures detected on the MDCs, and the green dashed lines correspond to the corrected calculated DFT bands. (b) MDCs extracted from the band mapping showing the $\mathrm{HH}, \mathrm{LH}$, and SO bands. MDCs are extracted every $-0.2 \mathrm{eV}$ between 0.0 and $-4.0 \mathrm{eV}$ (B.E.) and are vertically shifted for clarity. The arrows point to the MDCs associated with the $-1,-2,-3$, or $-4 \mathrm{eV}$ binding energies.

set of Luttinger parameters has been chosen to reproduce all experimental data.

In Fig. 3 we present the band structure along different $\mathbf{k -}$ space linear segments parallel to each other, with $\mathbf{k}_{\|}$along $\Delta$ in Fig. 3(a) or $\Sigma$ in Fig. 3(b), at various constant $\mathbf{k}_{\perp}$ values. For clarity, the split-off (SO) bands have been ignored, some sectors of the bands have been omitted in this illustration, and the Luttinger parameter $\gamma_{2}$ has been supposed to be zero. The same color in the two parts of Fig. 3 has been attributed to the same bands, and the holes appear either heavy or light depending on the direction, $\Delta$ or $\Sigma$.

When moving away from the $\Gamma$ point, the threefold degenerate band dispersing with momentum parallel to the $\Delta_{\mathrm{y}}$ direction split into two quasiparabolic LH bands, symmetric with respect to $\Gamma \Delta_{x}$ at all energies. The maxima (or tops) of the LH bands progressively spread and sink to lower binding energy. The top of the SO band passed through the crossing point of the LH bands and progressively sank to lower binding energy, following the purple arched curve in the $\left(\Gamma \Delta_{x} E\right)$ plane. The tops of the LH bands connected by the red arches were aligned along the $\Sigma$ direction and described the HH band of the $\Sigma$ axis.

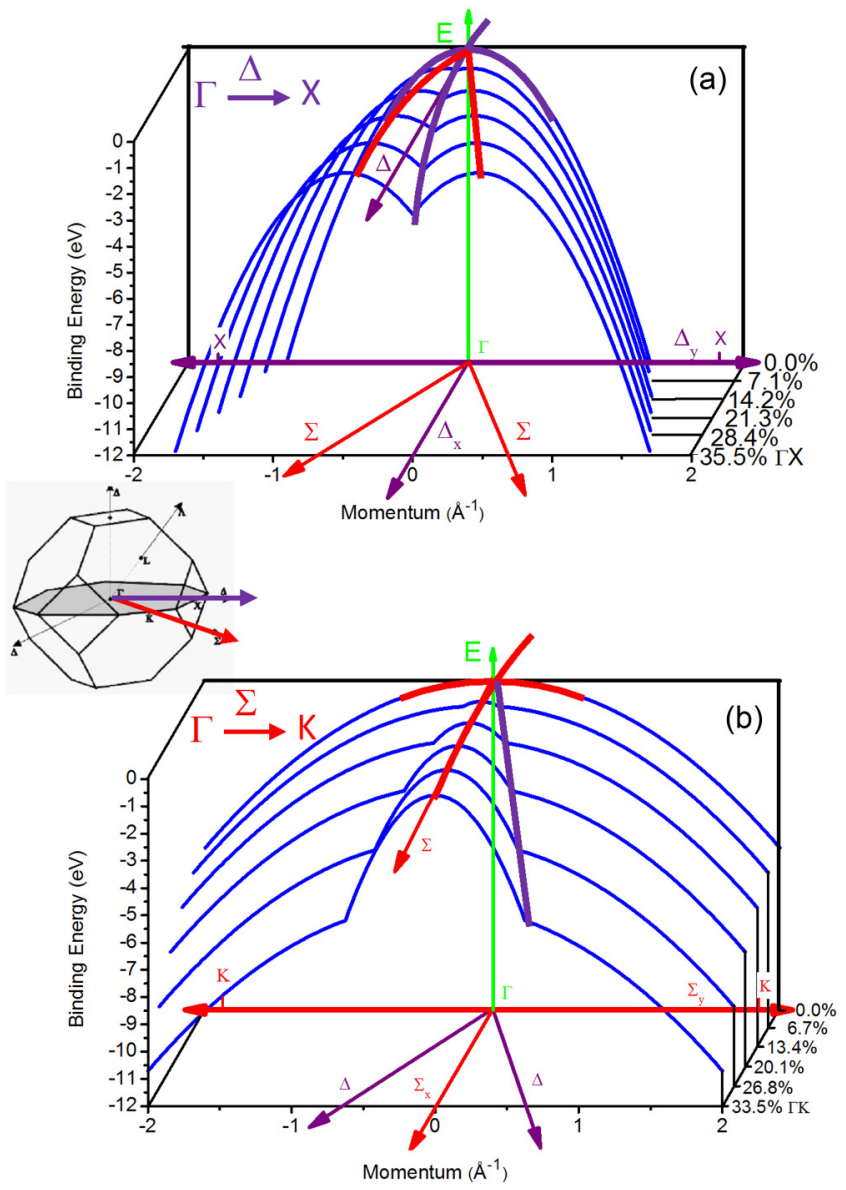

FIG. 3. (Color online) 3D evolution of the HH and LH bands calculated with the Luttinger parameters $\gamma_{1}=2.40, \gamma_{2}=0$, and $\gamma_{3}=$ 1.05 using the $\mathbf{k} \cdot \mathbf{p}$ model (a) along different directions parallel to $\Gamma X$ and (b) along different directions parallel to $\Gamma K$.

However, the nondegenerate bands dispersing with momentum parallel to the $\Sigma_{y}$ direction did not show any splitting. The tops of the bands sank to lower binding energy more or less rapidly and remained aligned with $\Gamma \Sigma_{x}$ at all energies.

\section{B. Determination of the Luttinger parameters}

In Figs. 4 and 5 we compare the model presented in Fig. 3(a) with experimental data collected parallel to the $\Gamma X$ direction but at different values of $\mathbf{k}_{\perp}$, in the third and second BZ, respectively. In Fig. 6 we show the same for the $\Gamma K$ direction of the second BZ. This was achieved by using photons of different energies ranging from 402 down to $355 \mathrm{eV}$ (third $\mathrm{BZ}$ ) and from 170 up to $200 \mathrm{eV}$ (second BZ), with an electron momentum $\mathbf{k}_{\|}$dispersing parallel to the $\Delta$ direction (Figs. 4 and 5) or to the $\Sigma$ direction (Fig. 6). The warped structure is clearly seen when ARPES experiments are performed away from the zone center $\left(\mathbf{k}_{\perp} \neq 0\right)$. Parallel to the $\Delta$ direction, the LH bands progressively split and moved to lower binding energy as the photon energy was decreased below $402 \mathrm{eV}$, but also, in a similar way, when the photon energy was increased (Fig. 4). This result clearly illustrated the evolution of the band structure, confirming the prediction of the $\mathbf{k} \cdot \mathbf{p}$ model. The third 


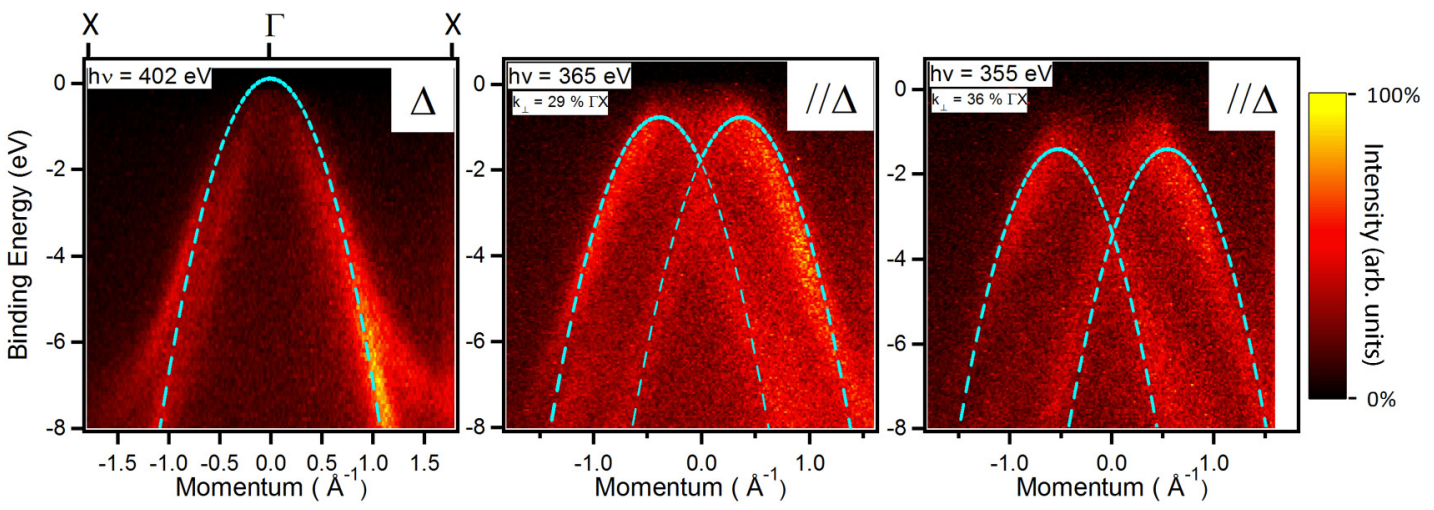

FIG. 4. (Color online) Mapping of the band structures measured parallel to the $\Gamma X$ direction, as a function of the photon energy (third BZ). The k.p model LH bands calculated with the most relevant set of Luttinger parameters (blue dashed line) are superimposed.

band was not detectable, probably due to the photoemission matrix element effects.

To determine experimentally and with good accuracy the Luttinger parameters that allowed us to build the pictures presented in Fig. 3, it was necessary to perform this extended band mapping away from the high-symmetry directions. The three band structures shown in Fig. 4 have been fitted with $\mathbf{k} \cdot \mathbf{p}$ bands using a unique set of parameters: $\gamma_{1}=2.40 \pm 0.05$, $\gamma_{2} \approx 0$, and $\gamma_{3}=1.05 \pm 0.05$.

While the situation was clear in the third BZ, the experimental signatures of the band structure behaved differently in the second BZ (Fig. 5). The bands degenerate at the $\Gamma$ point (i.e., $h v=170 \mathrm{eV}$ ) and progressively split into several bands that are not directly addressable using the $\mathbf{k} \cdot \mathbf{p}$ theory. This discrepancy can be explained by the surface sensitivity of the measurements performed at this lower photon energy. Nevertheless, even if we cannot clearly resolve the electronic bands, the broadening of these bands at $170 \mathrm{eV}\left[\Delta \mathbf{k}_{\|}=\right.$ $\left.0.42(5) \AA^{-1}\right]$ suggests a quite small value of the $\gamma_{2}$ parameter. On the contrary, we can clearly resolve the electronic bands measured parallel to the $\Sigma$ direction in this second BZ, as presented in Fig. 7. In this figure, the two lower bands appear on the band mapping with a good contrast, while the upper band, corresponding to the $\mathrm{HH}$ band, remains weak at all photon energies. This $\mathrm{HH}$ band is better revealed by the blue dots in Fig. 2(b) showing the stacked MDCs. Fitting the ARPES bands of Fig. 6 leads to an alternative determination of the
Luttinger parameters $\gamma_{1}=2.45, \gamma_{2} \approx 0.05$, and $\gamma_{3}=0.95$ that confirmed the previous values within the calculation accuracy (0.05). This set of parameters finally depicts the bands in the directions parallel to $\Delta$ and $\Sigma$.

As shown in Figs. 5 and 6 , the photoelectronic signal corresponding to the $\mathrm{HH}$ bands along the $\Sigma$ direction, but also that of the SO and the split LH bands along the $\Delta$ direction $(h v>170 \mathrm{eV})$, is weak in some areas. Some of these bands are the same seen from a different perspective, as illustrated by the $3 \mathrm{D}$ representation of the theoretical bands (Fig. 3). If the existence of the $\mathrm{HH}$ band becomes quite obvious using a stacked MDC presentation, the presence of the upper split LH bands along the $\Delta$ direction can be controlled in the same way or by changing the experimental configuration of the ARPES measurements. Figure 7 shows the band mapping of doped diamond recorded $2.5^{\circ}$ off the $\Delta$ direction, with a photon energy of $180 \mathrm{eV}$. In this configuration, the split LH bands acquired part of the $\mathrm{HH}$ character, and the $\mathrm{LH}$ bands became more intense. In addition, the structures of the energy distribution curves (EDCs) and MDCs pointed to by the blue arrows clearly indicated the presence of the split bands passing through the points $\left[\mathbf{k}_{\|}= \pm 0.47(5) \AA^{-1}\right.$ and $\mathrm{E}=-1.90 \mathrm{eV}$ (B.E.)].

We have seen that one set of Luttinger parameters fits all band structures measured parallel to the two main directions $\Delta$ and $\Sigma$ within the calculation accuracy. The value of $\gamma_{2}$ has a weak influence on the profile of the $\mathrm{HH}, \mathrm{LH}$, or SO bands over a broad binding energy range. If $\gamma_{2}=0.0$, the bands along $\Delta$ are

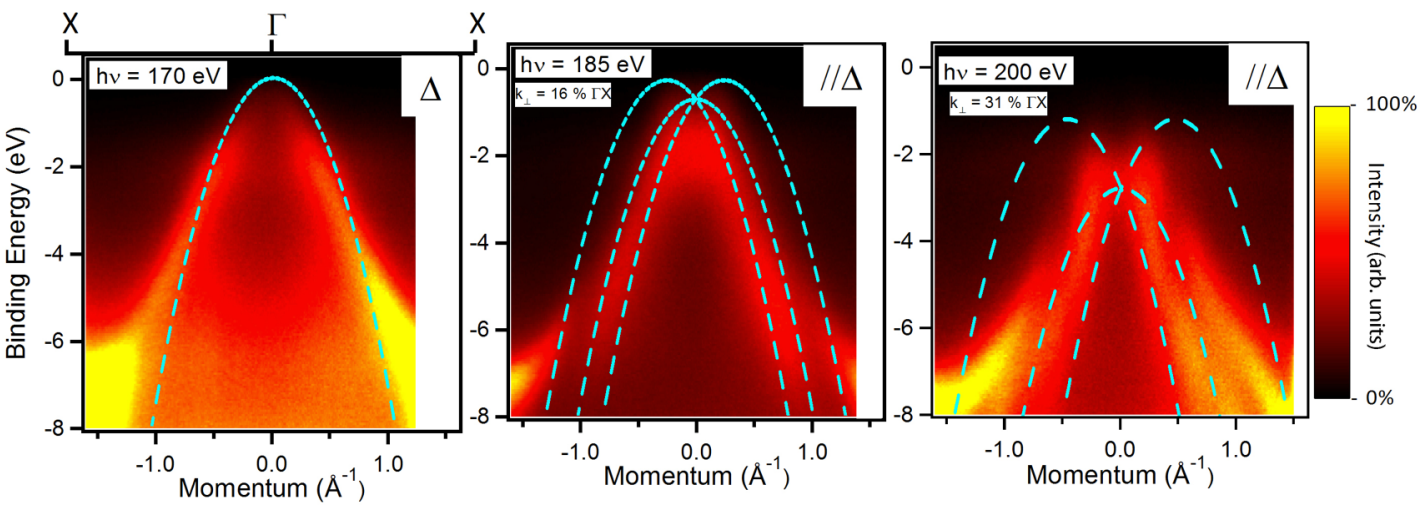

FIG. 5. (Color online) Mapping of the band structures measured parallel to the $\Gamma X$ direction, as a function of the photon energy (second BZ). The k·p model LH and SO bands calculated with the most relevant set of Luttinger parameters (blue dashed line) are superimposed. 

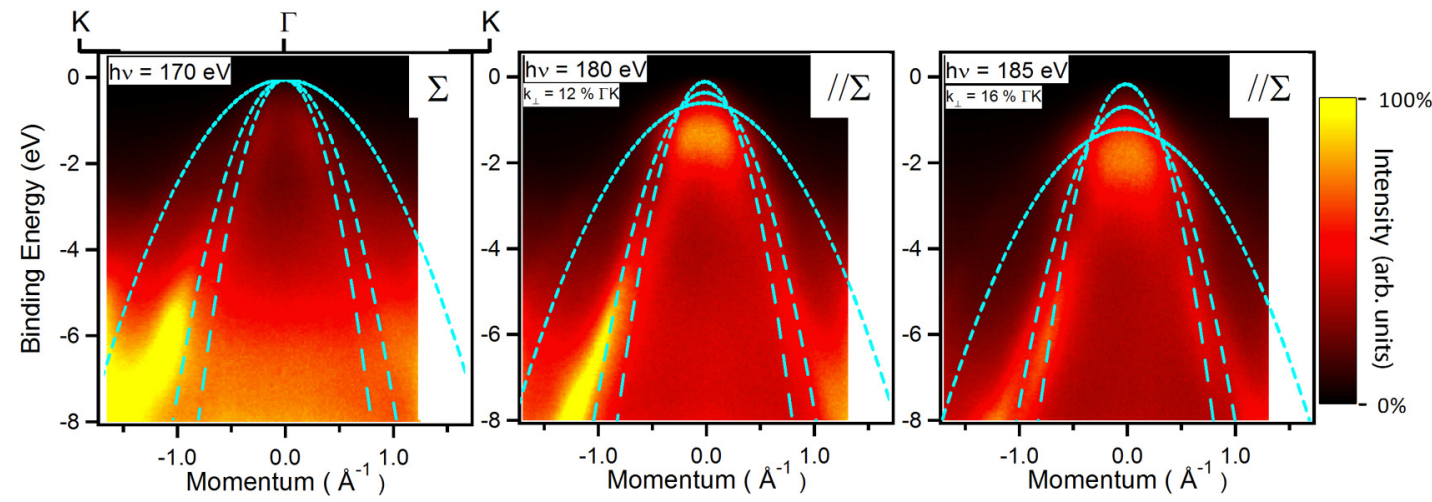

FIG. 6. (Color online) Mapping of the band structures measured parallel to the $\Gamma K$ direction, as a function of the photon energy (second BZ). The corrected DFT band (green dashed line) and $\mathbf{k} \cdot \mathbf{p}$ model HH, LH, and SO bands calculated with the most relevant set of Luttinger parameters (blue dashed line) are superimposed.

expected to be threefold degenerate, at variance with the DFT band structure, which involves one separate band and a twofold degenerate band. Experimentally, only one unresolved band is measured at the top of the band structure. A nonzero value for $\gamma_{2}$ would lift totally the degeneracy of the bands along $\Delta$, more consistent with the DFT band structure. Consequently, the two split LH bands, generated by the $\mathbf{k} \cdot \mathbf{p}$ model as soon as $\mathbf{k}_{\perp} \neq 0$, do not cross anymore; neither does the third SO band. The experimental analysis of band crossing may thus lead to estimating $\gamma_{2}$.

The crossings of the LH bands measured in the third or second BZ do not show any fingerprint of a gap at their intersection located at $\mathbf{k}_{\|}=0.0 \AA^{-1}$ (Figs. 4 and 5). More precisely, the analysis of the EDCs and MDCs demonstrated that the crossing of the LH bands at the point $\left(\mathbf{k}_{\|}=0 \AA^{-1}\right.$, B.E. $\left.=-1.90 \mathrm{eV}\right)$, for the band structure presented in the Fig. 7, corresponded to a maximum of ARPES signal, without any evidence for a gap opening. Nevertheless, the maximum in intensity would also be compatible with the presence of narrow gap filled with the third SO band passing through the center of the gap, as predicted by the k·p theory. Within experimental accuracy, the comparison of the profile of the MDC with the section of a LH band far away from any band intersection-typically $\Delta \mathbf{k}=0.42 \AA^{-1}$-allows us to conclude that $\left|\gamma_{2}\right|$ does not exceed 0.10 . This last observation confirmed the determination of a unique set of Luttinger parameters, $\gamma_{1}=2.40, \gamma_{2} \approx 0$, and $\gamma_{3}=1.00$, with a precision of \pm 0.05 , for describing the band structures of heavily doped diamond along many linear segments of the reciprocal space around the $\Gamma$ point.

\section{DISCUSSION}

\section{A. Luttinger parameters}

As summarized by Willatzen et al. [30], a large number of Luttinger parameter values have been published in the early literature about pure and semiconducting diamond. Most of them result from theoretical calculations based on the extended band structure, including several valence bands and empty electron bands, ranging from $\Gamma_{1}^{\mathrm{v}}$ to $\Gamma_{25}^{\mathrm{c}}$ points in the notation of Dresselhaus et al. [27]. The relatively wide dispersion of these values is generally attributed to the different methods of calculation that have been used. Experimental
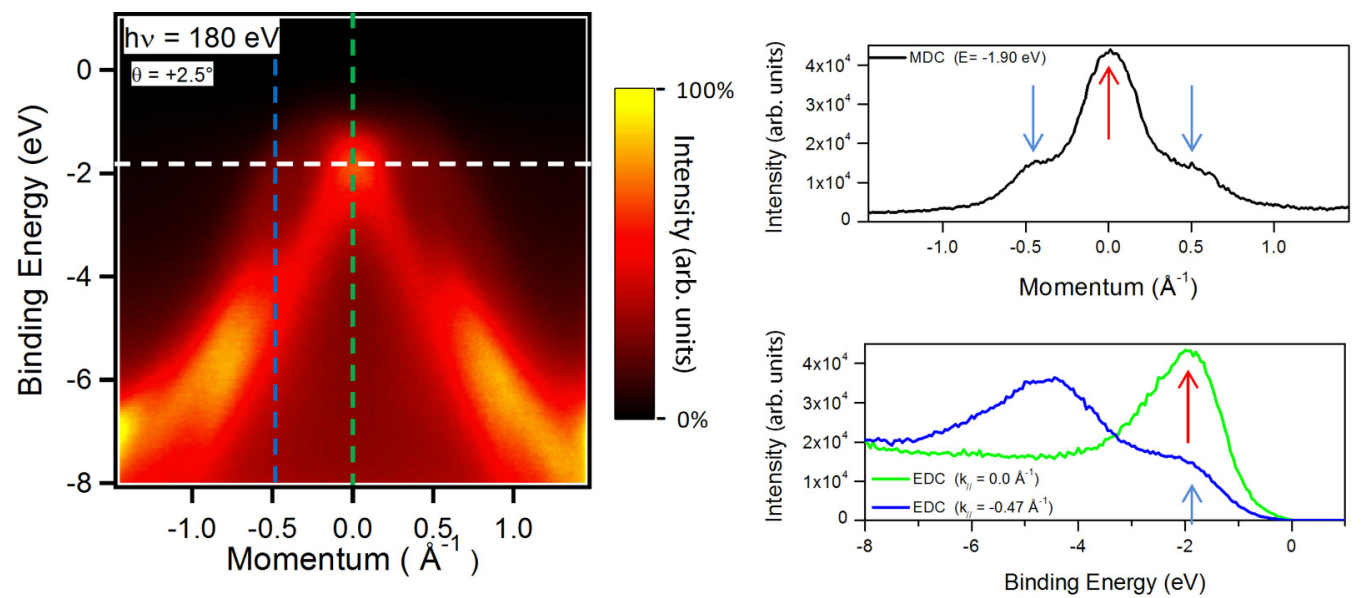

FIG. 7. (Color online) (left) Mapping of the band structure measured at $2.5^{\circ}$ off the $\Delta$ direction, close to the $\Gamma$ point $(h v=180 \mathrm{eV})$, and (right) $\mathrm{MDC}$ at $\mathrm{E}_{\mathrm{B}}=-1.90 \mathrm{eV}$ (black curve), $\mathrm{EDC}$ at $\mathbf{k}_{\|}=-0.47 \AA^{-1}$ (blue curve), and $\mathrm{EDC}$ at $\mathbf{k}_{\|}=-0.00 \AA^{-1}$ (green curve) extracted from the mapping. These curves attest the existence of the LH band at the crossing points $\left(\mathbf{k}_{\|}= \pm 0.47 \AA^{-1} ; \mathrm{E}_{\mathrm{B}}=-1.90 \mathrm{eV}\right.$, blue arrows) and the presence of a maximum of intensity at the bands' intersection $\left(\mathbf{k}_{\|}=0.0 \AA^{-1} ; \mathrm{E}_{\mathrm{B}}=-1.90 \mathrm{eV}\right.$, red arrows). 
TABLE I. Luttinger parameters for diamond determined in the metallic phase (present paper) and in the semiconducting phase.

\begin{tabular}{lccc}
\hline \hline & $\gamma_{1}$ & $\gamma_{2}$ & $\gamma_{3}$ \\
\hline Present paper & $2.40 ; 2.45$ & $0 ;-0.05$ & $1.05 ; 0.95$ \\
Willatzen et al. [30] & 2.54 & -0.10 & 0.63 \\
Bashenov et al. [46] & 2.19 & -0.12 & 0.87 \\
Kono et al. [45] & $0.67 ; 0.67$ & $-0.57 ;-0.98$ & $-2.23 ; 0.56$ \\
Naka et al. [26] & 2.67 & -0.40 & 0.68 \\
\hline \hline
\end{tabular}

Luttinger parameters for diamond were obtained by a cyclotron resonance technique in the semiconducting or pristine phase. The early determination proposed by Kono et al. [45] has been recently revised by Naka et al. [26]. Table I summarizes the most generally accepted values.

The present experimental determination of the Luttinger parameters, resulting from a study of the boron doped and metallic phase of diamond, appears relatively close to most theoretical or experimental values proposed recently for semiconducting diamond, especially for $\gamma_{1}$. Despite the difference of the phases, this agreement is not surprising: the band structure of metallic diamond has been considered quite similar to that of semiconducting diamond [5], and boron doping does not significantly change the band structure [31].

\section{B. Effective masses}

According to the k·p theory [29,30], the band masses along the high-symmetry directions can be derived from the Luttinger parameters using the following expressions:

$$
\text { along the } \Delta \text { direction : } \begin{aligned}
m_{1}^{-1} & =-\gamma_{1}-4 \gamma_{2} ; \\
m_{2}^{-1} & =m_{3}^{-1}=-\gamma_{1}+2 \gamma_{2}
\end{aligned}
$$

along the $\Sigma$ direction : $m_{1}^{-1}=-\gamma_{1}-\gamma_{2}-3 \gamma_{3}$;

$$
\begin{aligned}
& m_{2}^{-1}=-\gamma_{1}+2 \gamma_{2} ; \\
& m_{3}^{-1}=-\gamma_{1}-\gamma_{2}+3 \gamma_{3}
\end{aligned}
$$

The results are summarized in Table II. The leftmost values of this table (column $1, \gamma_{2}=0$ ) correspond to the five band masses calculated with the sets $\gamma_{1}=2.40, \gamma_{2}=0$, and $\gamma_{3}=1.05$ or $\gamma_{1}=2.45, \gamma_{2}=0$, and $\gamma_{3}=0.95$. The double values express the accuracy on the masses resulting from precision on the Luttinger parameters. This accuracy remains limited, and one can retain the values: $m_{\mathrm{hh}}^{100}=0.41(2)$, $m_{\mathrm{lh}}^{100}=0.41(2), m_{\mathrm{so}}^{100}=0.41(2), m_{\mathrm{lh}}^{110}=0.41(2)$, and $m_{\text {so }}^{110}=$ $0.18(5)$, according to the standard notations. A nonzero value for $\gamma_{2}$ modifies the lighter masses, as shown in column 3 for the limit case $\gamma_{2}=-0.10$. These mass values correspond to the LMTO masses calculated by Willatzen et al. (column 4) after an energy rescaling but are not corrected to take into account an adjusted spin-orbit coupling, which would slightly change theses values [30]. A direct extraction of the mass values by a parabolic curve fitting of the $\mathbf{k} \cdot \mathbf{p}$ dispersion curves along $\Delta$ and $\Sigma$ close to the band maxima yields a different result, tabulated in column 2 , with slightly heavier masses: $m_{\mathrm{hh}}^{100}=$ $0.53(7), m_{\mathrm{lh}}^{100}=0.53(7), m_{\mathrm{so}}^{100}=0.53(7), m_{\mathrm{hh}}^{110}=1.60(2)$, $m_{\mathrm{lh}}^{110}=0.52(6), m_{\mathrm{so}}^{110}=0.31(5)$. These can be compared to the effective masses directly measured by cyclotron resonance (column 5) and to the masses obtained from the rescaled DFT bands by a parabolic or nonparabolic curve fit according to Eq. (2). Taking into account the nonparabolicity of the LDA-DFT curves ( $\alpha$ varies from 0.05 to 1.09 , depending on the branch) improved the quality of the fit over a large energy range and led to effective masses $\sim 10 \%$ lighter than those found using the simpler parabolic fit $(\alpha=0)$ over a reduced $1.5 \mathrm{eV}$ energy range near the band maximum.

Along the $\Delta$ direction, the unresolved ARPES bands lead to attribute only one mass $m_{\mathrm{lh}}^{100}$ in this direction. Nevertheless, the experimental accuracy of the ARPES measurements suggests the possible existence of two distinct masses, not exceeding the values tabulated in column 3 . The latter description is in good agreement with the rescaled DFT bands results and with the rescaled LMTO mass calculations. Reflecting a weak spin-orbit coupling, the strong proximity of the two bands leads us to conclude that the two mass values lie close to each other, at variance with the recent direct measurements by cyclotron resonance that indicated the two masses would be significantly distant [26].

Along the $\Sigma$ direction, the present measurements by ARPES confirm the presence of the $\mathrm{HH}$ band with $m_{\mathrm{hh}}^{110} \approx$ 1.60 , a mass about three times heavier than its equivalent in the $\Delta$ direction. This value agrees with the calculations based on the LMTO, DFT, or $\mathbf{k} \cdot \mathbf{p}$ theories but diverges from the cyclotron resonance results performed in the 2 to $10 \mathrm{GHz}$ frequency range $[26,45]$. This discrepancy is resolved by using high-frequency cyclotron resonance at 70 or $150 \mathrm{GHz}$, which reveals several heavy masses $\left(1<m^{*}<2\right)$ [45,47].

\begin{tabular}{|c|c|c|c|c|c|c|c|}
\hline & $\begin{array}{c}\text { Present paper } \\
\text { Luttinger parameters } \\
\qquad \gamma_{2}=0\end{array}$ & $\begin{array}{c}\text { Present paper } \\
\mathbf{k} \cdot \mathbf{p} \text { curve fitting } \\
\gamma_{2}=0\end{array}$ & $\begin{array}{c}\text { Present paper } \\
\text { Luttinger parameters } \\
\gamma_{2}=-0.10\end{array}$ & $\begin{array}{l}\text { Willatzen et al. [30] } \\
\text { Corrected LMTO }\end{array}$ & $\begin{array}{c}\text { Naka et al. }[26] \\
\text { Cyclotron resonance }\end{array}$ & $\begin{array}{c}\text { Present paper } \\
\text { Rescaled DFT } \\
\text { Parabolic fit }\end{array}$ & $\begin{array}{c}\text { Present paper } \\
\text { Rescaled DFT } \\
\text { Nonparabolic fit }\end{array}$ \\
\hline$m_{\mathrm{hh}}^{100}$ & $0.417-0.408$ & 0.537 & 0.500 & 0.466 & 0.542 & 0.521 & 0.454 \\
\hline$m_{\mathrm{lh}}^{100}$ & $0.417-0.408$ & 0.537 & 0.385 & 0.366 & 0.288 & 0.345 & 0.320 \\
\hline$m_{\mathrm{so}}^{100}$ & $0.417-0.408$ & 0.537 & 0.385 & 0.366 & 0.288 & 0.345 & 0.320 \\
\hline$m_{\mathrm{hh}}^{110}$ & - & 1.602 & - & 1.783 & 0.700 & 1.593 & 1.427 \\
\hline$m_{\mathrm{lh}}^{110}$ & $0.417-0.408$ & 0.526 & 0.385 & 0.366 & 0.375 & 0.322 & 0.300 \\
\hline$m_{\mathrm{so}}^{110}$ & $0.180-0.189$ & 0.315 & 0.183 & 0.232 & 0.255 & 0.255 & 0.195 \\
\hline
\end{tabular}
The rejection of these values of masses as representative of impurities and not of the pure diamond may not apply to

TABLE II. Effective masses of the hole bands in diamond near the $\Gamma_{25}^{v}$ point. 
all cases, since it was demonstrated, by analyzing the few branches of the present band structure close to the $\Gamma_{25}^{v}$ point, that at least one of the heavy masses should be attributed to pure diamond.

The lighter masses leave less ground for discussion. The $m_{1 \mathrm{~h}}^{110}$ mass related to the SO band was found to be close to 0.32 , associated with a midpoint value -0.05 for $\gamma_{2}$, while the lightest $m_{2 \mathrm{lh}}^{110}$ mass related to the LH band was found to be close to 0.20 . These values are consistent with theoretical calculations and agree with the experimental mass values measured by cyclotron resonance most recently [26,45].

\section{CONCLUSION}

Despite the presence of the substitutional doping boron impurities, the band structure of metallic diamond was revealed to be similar to the electronic structure of semiconducting diamond, apart from a rigid band energy correction with respect to the Fermi level. The ARPES results confirmed the rescaled theoretical DFT bands of undoped diamond as a suitable band structure standard. Guided by the k·p model, we have determined from photoemission experiments, collected from a (100)-oriented epilayer, a unique set of Luttinger parameters describing the band structures of metallic diamond. This set, checked to be valid in many directions of the reciprocal space, includes a nonzero $\gamma_{2}$ parameter with an upper absolute value of 0.1 . These Luttinger parameters allowed an independent determination of the different band masses along the two main directions [100] and [110], in good agreement with recent theoretical or cyclotron resonancebased papers.

\section{ACKNOWLEDGMENTS}

The authors are grateful to J. Pernot for the help provided during the calculation of the $\mathbf{k} \cdot \mathbf{p}$ model band structures. The authors are also indebted to X. Blase for providing the DFT band structures.
[1] R. G. Cavell, S. P. Kowalczyk, L. Ley, R. A. Pollak, B. Mills, D. A. Shirley, and W. Perry, Phys. Rev. B 7, 5313 (1973).

[2] R. N. Euwena, D. L. Wilhite, and G. T. Suratt, Phys. Rev. B 7, 818 (1973).

[3] A. Mauger and M. Lannoo, Phys. Rev. B 15, 2324 (1977).

[4] F. J. Himpsel, J. F. van der Veen, and D. E. Eastman, Phys. Rev. B 22, 1967 (1980).

[5] B. Pate, Surf. Sci. 165, 83 (1986).

[6] J. R. Chelikowsky and S. G. Louie, Phys. Rev. B 29, 3470 (1984).

[7] J. Van der Weide and R. J. Nemanich, J. Vac. Sci. Tech. B 12, 2475 (1994).

[8] L. Diederich, O. M. Küttel, E. Schaller, and L. Schlapbach, Surf. Sci. 349, 176 (1996).

[9] F. Maier, R. Graupner, M. Hollering, L. Hammer, J. Ristein, and L. Ley, Surf. Sci. 443, 177 (1999).

[10] I. Jimenez, L. J. Terminello, D. G. J. Sutherland, J. A. Carlisle, E. L. Shirley, and F. J. Himpsel, Phys. Rev. B 56, 7215 (1997).

[11] E. A. Ekimov, V. A. Sidorov, E. D. Bauer, N. N. Mel'nik, N. J. Curro, J. D. Thompson, and S. M. Stishov, Nature 428, 542 (2004).

[12] E. Bustarret, J. Kačmarčik, C. Marcenat, E. Gheeraert, C. Cytermann, J. Marcus, and T. Klein, Phys. Rev. Lett. 93, 237005 (2004).

[13] T. Yokoya, T. Nakamura, T. Matsushita, T. Muro, Y. Takano, M. Nagao, T. Takenouchi, H. Kawarada, and T. Oguchi, Nature 438, 647 (2005).

[14] H. Okazaki, T. Yokoya, J. Nakamura, N. Yamada, T. Nakamura, T. Muro, Y. Tamenori, T. Matsushita, Y. Takata, T. Tokushima, S. Shin, Y. Takano, M. Nagao, T. Takenouchi, H. Kawarada, and T. Oguchi, J. Phys. Chem. Solid. 69, 2978 (2008).

[15] H. Okazaki, T. Wakita, T. Muro, T. Nakamura, Y. Muraoka, T. Yokoya, S. I. Kurihara, H. Kawarada, T. Oguchi, and Y. Takano, Appl. Phys. Lett. 106, 052601 (2015).

[16] P. Lawaetz, Phys. Rev. B 4, 3460 (1971).
[17] H. Kim, R. Vogelgesang, A. K. Ramdas, S. Rodriguez, M. Grimsditch, and T. R. Anthony, Phys. Rev. B 57, 15315 (1998).

[18] V. N. Denisov, B. N. Mavrin, S. N. Polyakov, M. S. Kuznetsov, S. A. Terentiev, and V. D. Blank, Phys. Lett. A 376, 2812 (2012).

[19] J. Serrano, M. Cardona, and T. Ruf, Solid State Comm. 113, 411 (2000).

[20] M. Cardona, T. Ruf, and J. Serrano, Phys. Rev. Lett. 86, 3923 (2001).

[21] R. Sauer, H. Sternschulte, S. Wahl, K. Thonke, and T. R. Anthony, Phys. Rev. Lett. 84, 4172 (2000).

[22] R. Sauer and K. Thonke, Phys. Rev. Lett. 86, 3924 (2001).

[23] E. Gheeraert, S. Koizumi, T. Teraji, H. Kanda, and M. Nesladek, Phys. Status Solidi 174, 39 (1999).

[24] L. Reggiani, D. Waechter, and S. Zukotynski, Phys. Rev. B 28, 3550 (1983).

[25] H. Löfås, A. Grigoriev, J. Isberg, and R. Ahuja, AIP Adv. 1, 032139 (2011).

[26] N. Naka, K. Fukai, Y. Handa, and I. Akimoto, Phys. Rev. B 88, 035205 (2013).

[27] G. Dresselhaus, A. F. Kip, and C. Kittel, Phys. Rev. 98, 368 (1955).

[28] P. Y. Yu and M. Cardona, Fundamentals of Semiconductors, 2nd ed. (Springer-Verlag, Berlin, 1999), p. 63.

[29] K. Y. Shtivel'man, Sov. Phys. Solid State 5, 252 (1963).

[30] M. Willatzen, M. Cardona, and N. E. Christensen, Phys. Rev. B 50, 18054 (1994).

[31] X. Blase, Ch. Adessi, and D. Connétable, Phys. Rev. Lett. 93, 237004 (2004).

[32] H. J. Xiang, Z. Li, J. Yang, J. G. Hou, and Q. Zhu, Phys. Rev. B 70, 212504 (2004).

[33] F. Giustino, J. R. Yates, I. Souza, M. L. Cohen, and S. G. Louie, Phys. Rev. Lett. 98, 047005 (2007).

[34] K. W. Lee and W. E. Pickett, Phys. Rev. B 73, 075105 (2006). 
[35] T. Shirakawa, S. Horiuchi, Y. Ohta, and H. Fukuyama, J. Phys. Soc. Jpn. 76, 014711 (2007).

[36] S. L. Chuang, Phys. Rev. B 43, 9649 (1991).

[37] T. Wojewoda, P. Achatz, L. Ortéga, F. Omnès, C. Marcenat, E. Bourgeois, X. Blase, F. Jomard, and E. Bustarret, Diam. Rel. Mater. 17, 1302 (2008).

[38] A. Kawano, H. Ishiwata, S. Iriyama, R. Okada, T. Yamaguchi, Y. Takano, and H. Kawarada, Phys. Rev. B 82, 085318 (2010).

[39] P. Achatz, F. Omnès, L. Ortéga, C. Marcenat, J. Vacík, V. Hnatowicz, U. Köster, F. Jomard, and E. Bustarret, Diam. Rel. Mater. 19, 814 (2010).

[40] S. Hüfner, Photoelectron Spectroscopy, Vol. 82 (SpringerVerlag, Berlin, 1995).
[41] J. M. Soler, E. Artacho, J. D. Gale, A. Garc, J. Junquera, P. Ordej, and D. Sanchez-Portal, J. Phys. Condens. Matter 14, 2745 (2002).

[42] J. M. Luttinger, Phys. Rev. 102, 1030 (1956).

[43] M. S. Hybertsen and S. G. Louie, Phys. Rev. B 34, 5390 (1986).

[44] X. Blase, Phys. Rev. B 67, 035211 (2003).

[45] J. Kono, S. Takeyama, T. Takamasu, N. Miura, N. Fujimori, Y. Nishibayashi, T. Nakajima, and K. Tsuji, Phys. Rev. B 48, 10917 (1993).

[46] V. K. Bashenov, A. G. Gontar, and A. G. Petukhov, Phys. Status Solidi B 108, K139 (1981).

[47] C. J. Rauch, Phys. Rev. Lett. 7, 83 (1961). 